\title{
A Simulation for a Carbon Star
}

\section{GÜLÇINN KANDEMİR and CEM GÜÇLÜ}

\author{
Physics Department, Istanbul Technical University \\ Istanbul, Turkey
}

\begin{abstract}
A simple one-dimensional electrostatic plasma simulation code has been applied at a carbon star to represent possible instabilities. Electrons are assumed to move against a stationary ion background. The temperature in the vicinity of the carbon star is adopted as $5,000-10,000 \mathrm{~K}$ and some plasma input parameters are calculated accordingly for this cold plasma. Here, the Maxwell and Vlasov equations are employed and the fast Fourier transform is applied in the program. Linear and non-linear effects resulting from plasma instabilities in carbon-star atmospheres have been investigated. Among the several types of plasma instability, electrostatic instability is the most destructive. Recently, the role of ionization is adopted as 10 times more in the carbon stars. The electrostatic instability grows by accumulation of charge and the perturbations grow exponentially with time. At a carbon-star plasma where large changes occur, the electrostatic instability should not be ignored. The present simulation shows that even small perturbations may cause instability in the carbon-star envelope. The calculated drift velocity found by this simulation is comparable to the velocity of $20 \mathrm{~km} \mathrm{~s}^{-1}$ indicated by recent observations of the expanding envelopes of carbon stars. In addition to the expected linear behavior, the non-linear behavior in phase space found by this simulation agrees with the theory.
\end{abstract}

